\title{
Hard Switching Characteristics of SiC and GaN Devices for Future Electric Vehicle Charging Stations
}

\author{
Gowthamraj Rajendran ${ }^{1}$, Chockalingam Aravind Vaithilingam ${ }^{1 *}$, Kanendra $\mathrm{Naidu}^{2}$, \\ Kameswara Satya Prakash ${ }^{1}$, and Md Rishad Ahmed ${ }^{3}$ \\ ${ }^{1}$ High Impact Research Lab, School of Engineering, Taylor's University Lakeside Campus, Subang \\ Jaya, Selangor, Malaysia, 47500. \\ ${ }^{2}$ Department of Electrical Technology, Universiti Kuala Lumpur British Malaysian Institute, Gombak \\ Selangor, Malaysia, 50250. \\ ${ }^{3}$ Electrical and Electronic Engineering, University of Nottingham, A61a, Coates Building, University \\ Park, Nottingham, NG7 2RD.
}

\begin{abstract}
Wide bandgap (WBG) semiconductors offer better switching and lower losses, and it is not uncommon to utilize them for high power density, high-efficiency applications. Gallium nitride $(\mathrm{GaN})$ and Silicon carbide $(\mathrm{SiC})$ are the most common WBG materials that are responsible for major switching level changes relative to silicon $(\mathrm{Si})$ devices. This paper explores the contrast of performance between $\mathrm{Si}, \mathrm{SiC}$, and $\mathrm{GaN}$ devices. The output performance of $\mathrm{Si}, \mathrm{SiC}$, and $\mathrm{GaN}$ power devices includes efficiency, energy bandgap, thermal conductivity, carrier mobility, saturation speed, power density, switching characteristics, and conduction losses. This article also proposes a Vienna rectifier with GaN materials, which operates as a front-end rectifier on a high-density battery charger targeted at highperformance applications such as electric vehicle charging stations, aircraft applications, and welding power sources. The system would reduce the total harmonics distortion (THD) to less than $5 \%$, and the power factor would be increased to unity to satisfy the IEEE-519 standard.
\end{abstract}

\section{Introduction}

A three-phase three-level Vienna rectifier has significant advantages compared to any other three-level power factor correction (PFC) rectifier in terms of improved power factor at the input side of the system, reduced power consumption of switches, and reduced overall input current harmonics distortion as one of the three-level boost converter clamping system [1].

The Vienna rectifier topology would effectively improve the power density of the system, particularly for high power applications such as electric vehicle charging stations, aircraft

\footnotetext{
* Corresponding author: aravindcv@ieee.org
} 
applications, and welding power sources, as shown in Fig. 1. The Vienna rectifier is the best suitable three-phase boost converter for the electric vehicle charging stations [2]; however, the input current harmonics distortion caused by the semiconductor switching operation can be very troublesome for the converters with the switching frequency of hundreds of $\mathrm{kHz}$, particularly near the zero-crossing phase current. The current harmonics distortion of the Vienna rectifier is mostly attributed to the super-junction MOSFET's non-linear output capacitance $C_{o s s}$, as the voltage rise at turn-off may be very long within a comparatively short period [3]. This creates an effective voltage error on the ac terminal of the power converter and subsequent distortion of the current harmonics in the input boost inductor. The $\mathrm{Si}$ MOSFET super junction has a considerably non-linear output capacitance, with a steep drop off higher orders of 50-100V. Initially, the slew rate of turn-off voltage is too poor before the drain-source voltage exceeds a higher order magnitude level. Hartmann et al. observed a substantial turn-off delay and comparatively low voltage rise time. They suggested a curve fitting of experimental data around the existing spectrum to balance the non-linear turn-off delay time [3]. However, the turn-off time of the gallium nitride $(\mathrm{GaN})$ enhancement (emode) device is significantly less [4-6]. Consequently, in the power electronic converter systems, various types of power semiconductor devices are implemented to reduce the turnoff time and voltage rise.
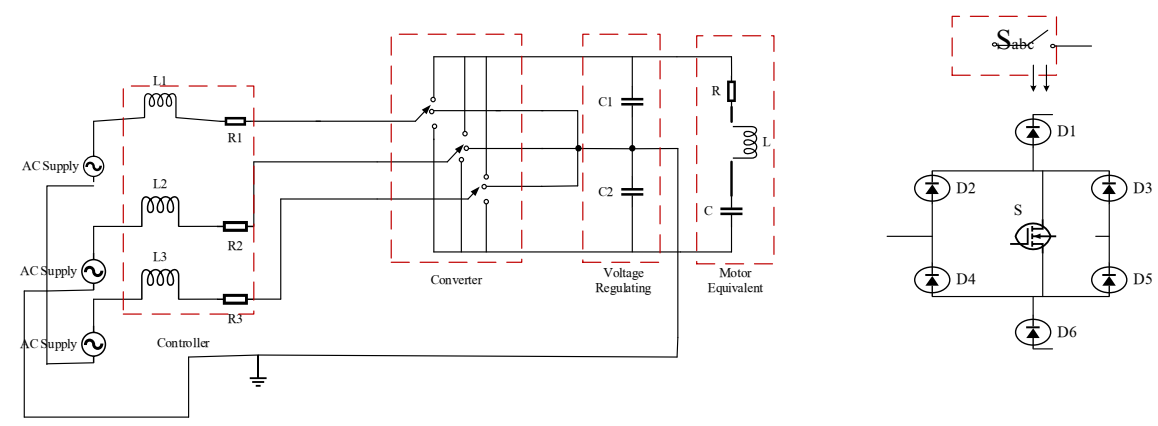

(a) Vienna configuration used in this study

(b) Converter

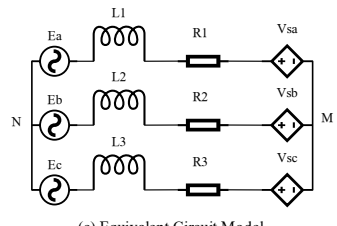

(c) Equivalent Circuit Model

Fig 1. Vienna Rectifier Topology [2]

Advancements in power semiconductor devices have enabled recent developments in power electronic systems operating at radio frequencies (RF) in device technologies, circuit configurations, and circuit design techniques [7]. The growing demand for electrical energy in various applications in the space industry and aircraft industry requires lightweight, efficient, and high power density switched-mode power supplies(SMPS) [8]. The GaN highelectron-mobility-transistors (HEMTs) have been successful candidates for size reduction, with improved high switching frequency and high performance. The high breakdown voltage of $\mathrm{GaN}$ has already allowed remarkable high-voltage applications [9]. These devices offer the lowest $R_{D S \text { (on) }}$ for a given voltage rating [10]. Furthermore, GaN epitaxial growth of large diameter $\mathrm{Si}$ substrates with new technology to deposit metal-organic chemical vapour allows GaN devices with high efficiency at low production cost in addition [11-14]. The GaN devices have effectively substituted Si devices in many applications such as welding power 
sources, electric vehicle charging stations [15], aircraft applications, telecommunication [16], and solar applications [17]. The silicon power semiconductor devices are used for high power applications with an operating frequency range between $10 \mathrm{~Hz}$ to $10 \mathrm{kHz}$, as shown in Fig. 2. The silicon-carbide power semiconductor devices are used for medium power applications with an operating frequency range between $10 \mathrm{kHz}$ and $10 \mathrm{MHz}$. The GaN devices are used for medium and low power applications with an operating frequency range between $100 \mathrm{kHz}$ to $100 \mathrm{MHz}$.

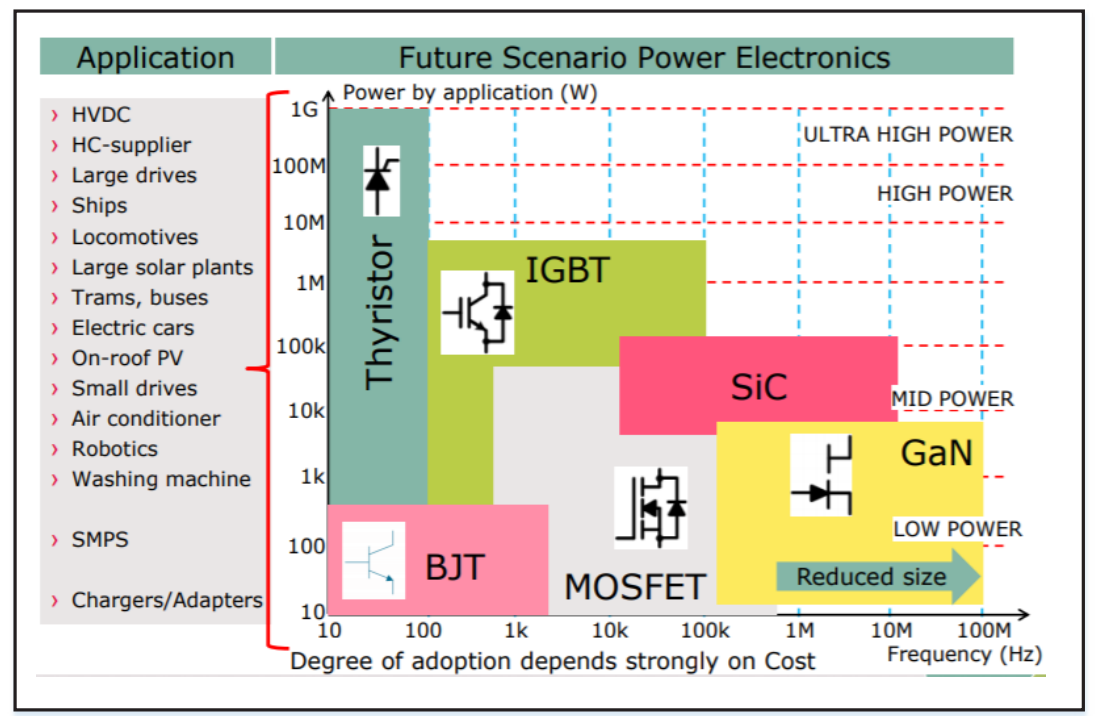

Fig 2. Potential Applications of Power Semiconductor Devices [9]

The silicon carbide power semiconductor devices are used for medium and high power applications such as welding power sources, telecommunication power sources, electric vehicle charging stations. The applications of $\mathrm{SiC}$ devices can be found for power factor correction converters such as interleaved PFC converter, three-phase B6 PFC converter, Vienna rectifier, classic PFC converter, Bridgeless PFC converter, as shown in Fig. 3. It is also used for interleaved LLC DC/DC converter, half-bridge LLC DC/DC converter, and solar string inverter applications. 


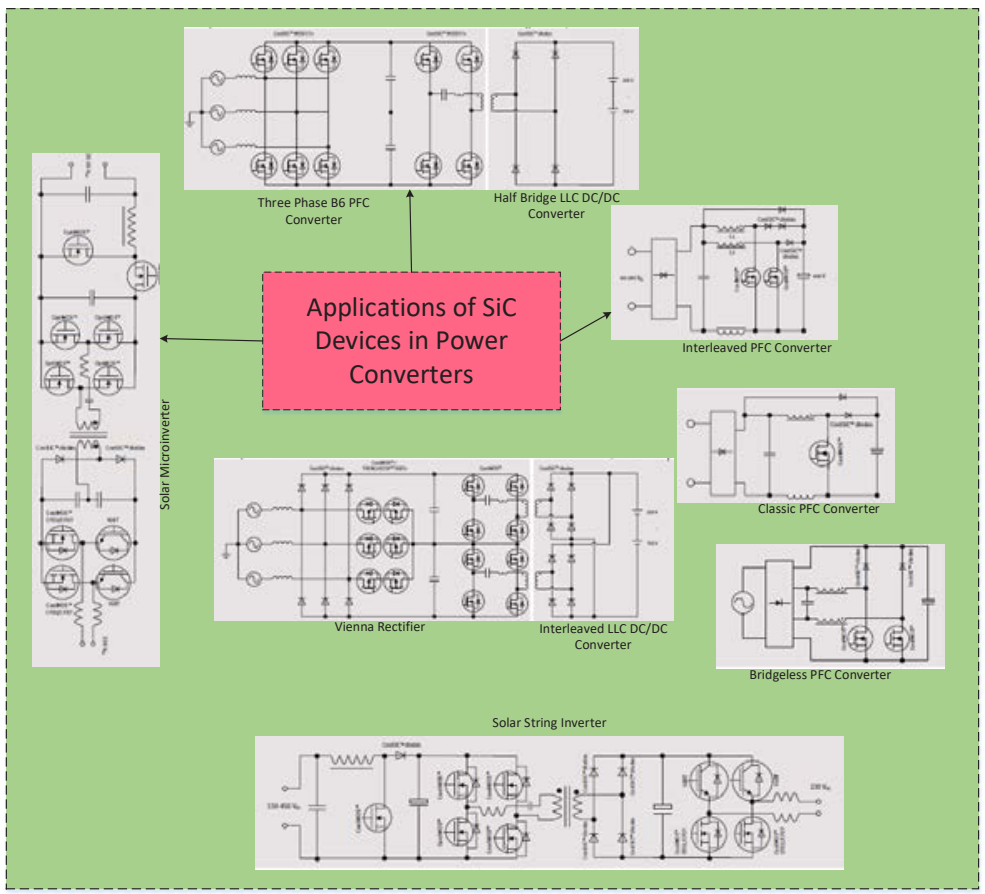

Fig 3. Applications of SiC devices in Power Converters [18]

The power density of the $\mathrm{SiC}$ system is high compared to Si-based systems due to the high switching frequency of the $\mathrm{SiC}$ semiconductor devices, resulting in improved efficiency of the system. The growth of $\mathrm{GaN}$ devices is expected to supersede the $\mathrm{SiC}$ devices in the market by 2030, as shown in Fig. 4.

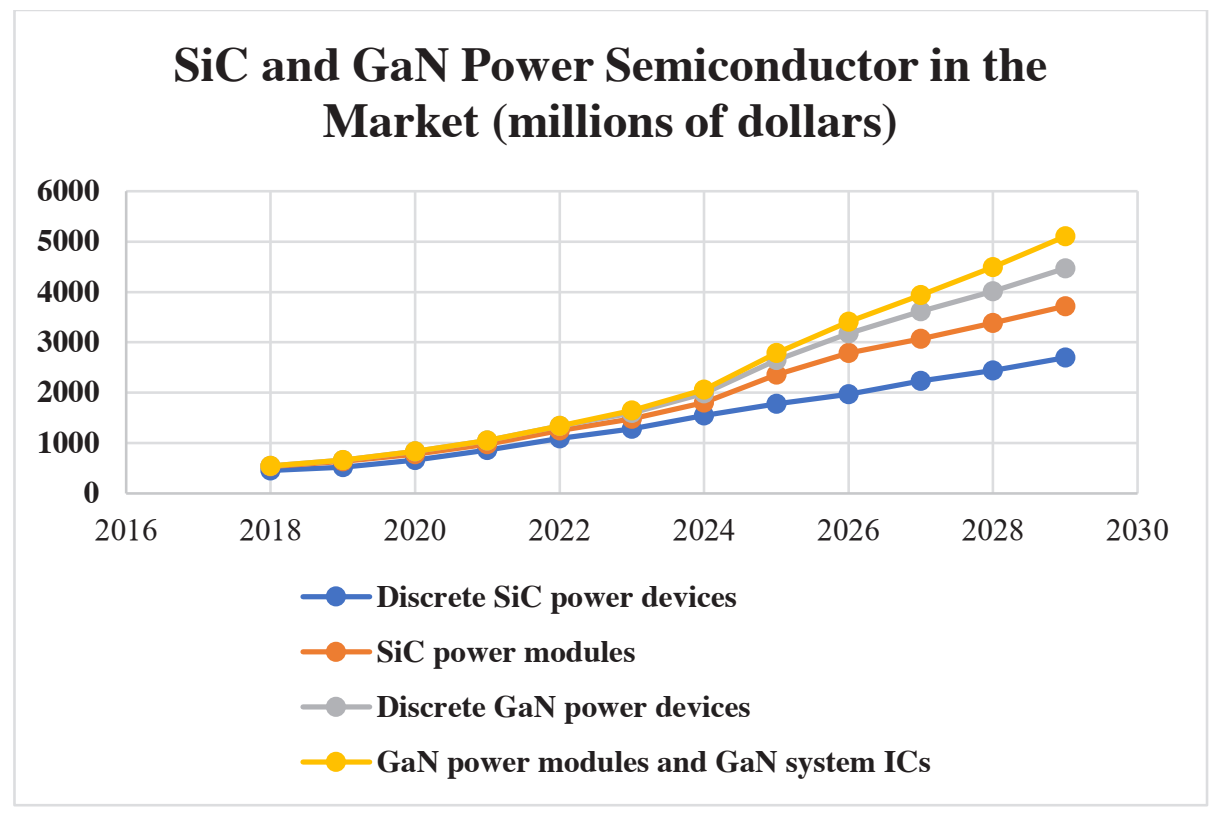

Fig 4. Comparison of $\mathrm{SiC}$ and $\mathrm{GaN}$ Devices in the Market [18] 
The efficiency, power density, thermal conductivity, energy band gap, carrier mobility, saturation velocity, switching characteristics, and conduction losses are analysed in this paper for $\mathrm{Si}, \mathrm{SiC}$, and $\mathrm{GaN}$ devices. This paper also proposes a unique power device for the Vienna rectifier based electric vehicle charging stations.

\section{Simulation of Temperature-Dependent Characteristics}

The performance of the $\mathrm{SiC}$ and $\mathrm{GaN}$ devices is addressed on the basis of temperature dependence, and the energy gap, carrier mobility, and saturation speed are contrasted.

\subsection{Energy band gap}

Dependent on the bandgap, the conductivity of the semiconductor is high. According to Varshini's equation, the energy gap is influenced by temperature [9].

$$
\begin{aligned}
& E_{g}(T)=E_{g}(0)-\frac{\alpha_{E} T^{2}}{T+\beta_{E}} \\
& E_{g}(0)=\text { Energy bandgap at } 0 \mathrm{~K} \\
& \alpha_{E} \text { and } \beta_{E}=\text { material constants }
\end{aligned}
$$

\begin{tabular}{|c|c|c|c|}
\hline Materials & $\mathrm{SiC}$ & GaN & $\mathbf{S i}$ \\
\hline Bandgap (eV) & 3.4 & 3.27 & 1.12 \\
\hline Breakdown field (MV/cm) & 3.0 & 4.9 & 0.3 \\
\hline Drift velocity $\left(10^{7} \mathrm{~cm} / \mathrm{s}\right)$ & $1-2.5(\mathrm{e})$ & 2.0 & 1 \\
\hline Carrier mobility $\left(\mathrm{cm}^{2} / \mathrm{V}-\mathrm{s}\right)$ & $2000(\mathrm{e})$ & 1000 & 1450 \\
\hline Dielectric Constant $\xi_{r}$ & 8.9 & 9.7 & 11.7 \\
\hline $\begin{array}{l}\text { Thermal conductivity (W/cm- } \\
\mathrm{K})\end{array}$ & 2 & 4.9 & 1.5 \\
\hline Relative Permittivity & 9.7 & 10.4 & 11.9 \\
\hline $\begin{array}{c}\text { Junction Temperature - Max } \\
\left({ }^{\circ} \mathrm{C}\right)\end{array}$ & 175 & 175 & 150 \\
\hline Switching Frequency & $>50 \mathrm{kHz}$ & $>10 \mathrm{MHz}$ & $<20 \mathrm{kHz}$ \\
\hline Gate charge $\left(\boldsymbol{Q}_{\boldsymbol{g}}\right)$ & $\begin{array}{l}\text { Slightly lower than } \\
\qquad \mathrm{Si}\end{array}$ & Lowest & Highest \\
\hline Threshold Voltage $\left(\boldsymbol{V}_{\boldsymbol{t h}}\right)$ & $3 \mathrm{~V}$ & $1.2-1.6 \mathrm{~V}$ & $3 \mathrm{~V}$ \\
\hline Maximum Vgs & $20 \mathrm{~V}$ & $7-10 \mathrm{~V}$ & $20 \mathrm{~V}$ \\
\hline $\begin{array}{c}\text { Drain to Source } \\
\text { leakage current in OFF state }\end{array}$ & Lowest & Highest & $\begin{array}{l}\text { Slightly Higher } \\
\text { than } \mathrm{SiC}\end{array}$ \\
\hline Body Diode $\left(\boldsymbol{V}_{f}\right)$ & $\sim 3.5 \mathrm{~V}$ & No body diode & $\sim 0.9 \mathrm{~V}$ \\
\hline Reverse Recovery $\left(\boldsymbol{Q}_{r r}\right)$ & $\begin{array}{l}\text { Slightly Higher than } \\
\text { GaN }\end{array}$ & Lowest & Highest \\
\hline Dynamic Rds_on & None & None & Up to $30 \%$ \\
\hline Most suitable for applications & $\begin{array}{c}\text { Forward, half-bridge } \\
\text { based topologies }\end{array}$ & $\begin{array}{l}\text { Half-bridge based } \\
\text { topologies }\end{array}$ & $\begin{array}{c}\text { Boost PFC, } \\
\text { flyback converter } \\
\text { topology }\end{array}$ \\
\hline
\end{tabular}

Table 1. Energy Band-Gap Parameters [19] 
Table 1 shows the constant values for specified materials. The bandgap value of the GaN device is $3.40 \mathrm{eV}$, which is higher than the bandgap of the $\mathrm{Si}$ and $\mathrm{SiC}$ device bandgaps of 1.1 $\mathrm{eV}$ and $3.26 \mathrm{eV}$. Fig. 5 indicates the temperature and energy bandgap relationship of GaN and $\mathrm{SiC}$ materials. The $\mathrm{GaN}$ devices have a high bandgap than the $\mathrm{SiC}$ device at room temperature. The switching frequency of the GaN device is high compared to $\mathrm{Si}$ and $\mathrm{SiC}$ devices, which leads to a small filter size of the power converter. Even though the input current harmonics of the GaN-based power converter is high due to the high switching frequency, the proposed GaN-based Vienna rectifier with the PFC controller can improve the reduce the harmonics are less than 5\% to satisfy the IEEE-519 Standard [20]-[21].

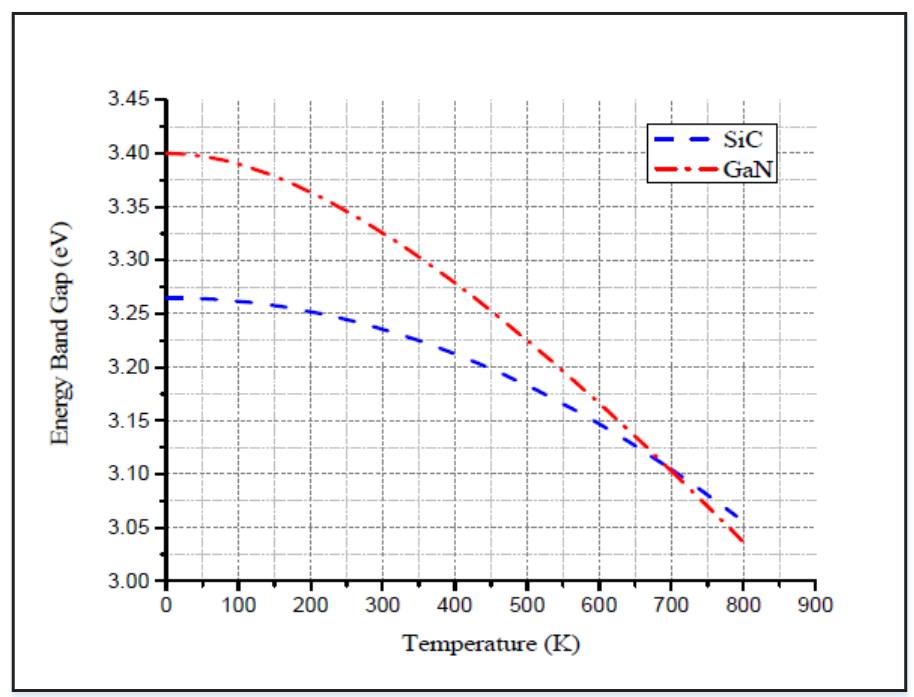

Fig 5. Temperature vs. Energy Bandgap for $\mathrm{SiC}$ and $\mathrm{GaN}[22]$

\subsection{Carrier mobility}

Carrier mobility is one of the significant constraints of MOSFET. The semiconductor material's conductivity is specifically based on integrating carrier concentration and associated mobility. The Bose-Einstein distribution theorem states that phonon scattering is extremely temperature-dependent. Increased phononic density, as with the rise in temperature, which increases scattering. This scattering contributes to the low mobility of carriers at high temperatures. The temperature-carrier relationship is indicated in Eq. 2 [22].

$$
\begin{aligned}
& \mu_{v}=\mu_{v 300 K} \cdot\left(\frac{T_{L}}{300 K}\right)^{\gamma} \\
& T_{L}=\text { variable temperature } \\
& \gamma=\text { constant }
\end{aligned}
$$$$
\mu_{v 300 K}=\text { carrier mobility at room temperature }
$$ 


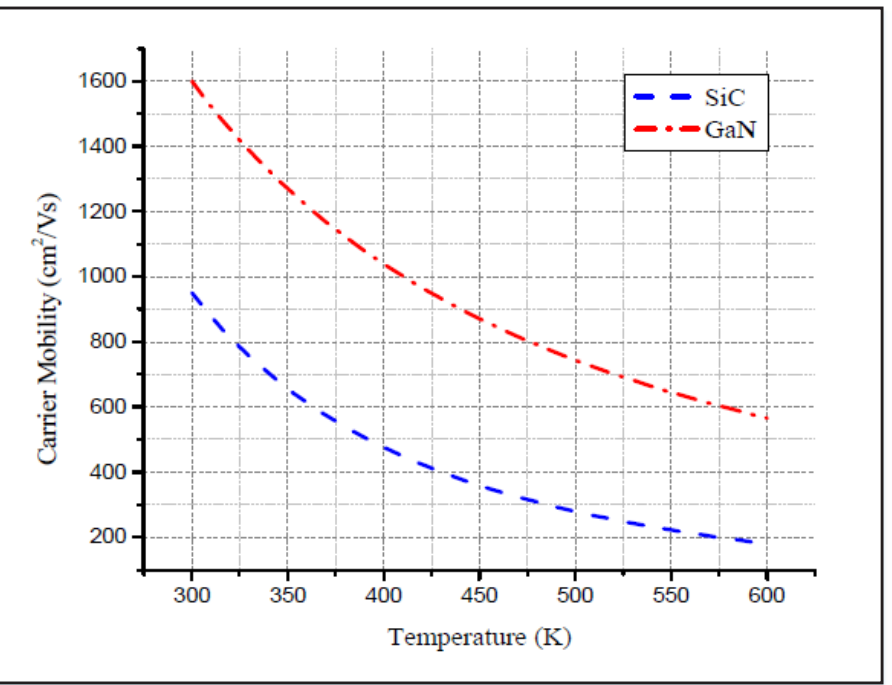

Fig 6. Temperature vs. carrier mobility for $\mathrm{SiC}$ and $\mathrm{GaN}[23]$

$\mathrm{GaN}$ offers improved electron mobility in competition with $\mathrm{Si}$ and $\mathrm{SiC}$ devices, proving that $\mathrm{GaN}$ is the best suitable semiconductor device for high-frequency applications. Fig. 6 demonstrates that $\mathrm{SiC}$ and $\mathrm{GaN}$ device carrier velocity is 950 and $1600 \mathrm{~cm}^{2} / \mathrm{Vs}$ at room temperature.

\subsection{Saturation velocity}

The gain is commensurate with the saturation velocity on the high-end devices or HEMTs. The carrier velocity achieves its optimal value by adding a large electric field. In addition, increased fields lead to energy loss by lattice interaction. These interactions contribute to the release of phonons and photons, which raises system temperature, possibly impacting the overall efficiency of the system. Eq.(3) describes the relationship between saturation velocity and temperature.

$$
\begin{aligned}
& V_{S A T}\left(T_{L}\right)=\frac{V_{S a t 300}}{\left(1-A_{n}\right)+A_{n} \frac{T_{L}}{300 K}} \\
& V_{\text {sat } 300}=\text { saturation velocity at room temperature } \\
& A_{n}=\text { temperature coefficient of the material } \\
& T_{L}=\text { variable temperature }
\end{aligned}
$$

The relative permittivity of $\mathrm{SiC}$ and $\mathrm{GaN}$ is low, and the saturation velocity of $\mathrm{SiC}$ and $\mathrm{GaN}$ is high compared to $\mathrm{Si}$ devices. The $\mathrm{SiC}$ materials have a $2 \times 10^{7} \mathrm{~cm} / \mathrm{s}$ saturation velocity while the GaN materials have $2.2 \times 10^{7} \mathrm{~cm} / \mathrm{s}$ saturation velocity and $\mathrm{SiC}$ and $\mathrm{GaN}$ temperature coefficient of 0.15 and 0.5 , respectively. The saturation velocity of all semiconductor materials is most significant in the GaN, as seen in Fig. 7. GaN devices can transfer a higher current density as electrons have a large saturation velocity in GaN. A higher current density transmission has the effect of improving the power density of the power 
converter, which results in a reduction in input and output filters. This means that the losses are minimized, and the system efficiency will be improved.

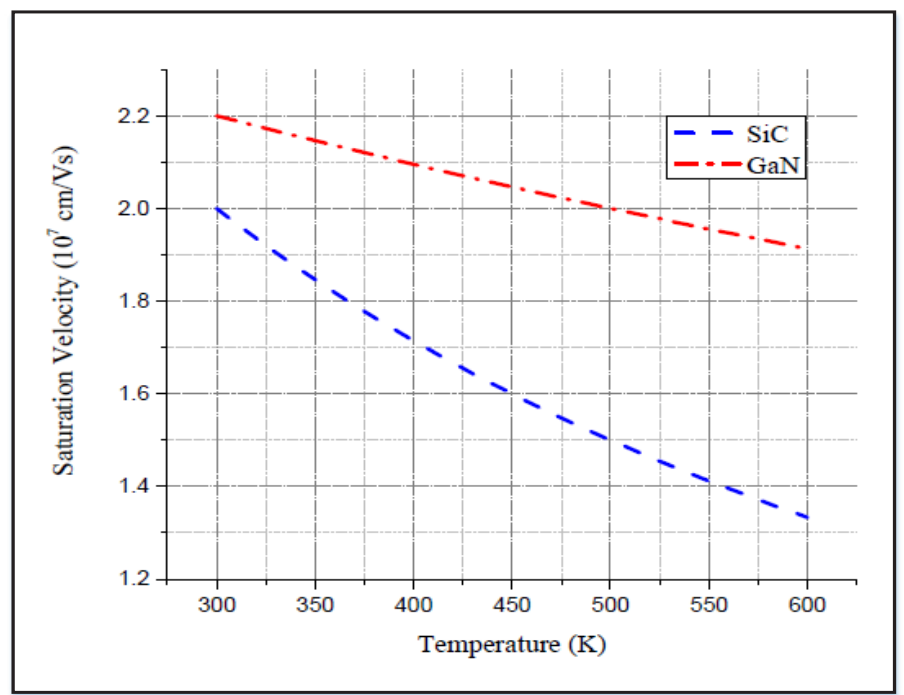

Fig 7. Saturation Velocity vs. Temperature [23]

\subsection{Evaluation of switching losses and conduction losses}

In this section, the conduction and switching losses were examined based on the types of power semiconductor devices. The conduction losses are measured using on-state resistance calculation and losses induced by the power semiconductor current depending on the junction temperature. The loss evaluation for switching frequencies of $10 \mathrm{kHz}$ and $100 \mathrm{kHz}$ for Si, $\mathrm{SiC}$, and $\mathrm{GaN}$ is seen in Fig. 8 and Fig. 9, respectively. GaN has the lowest losses at low junction temperature and low switching frequencies. The overall switching losses of GaN devices are smaller than the switching losses of $\mathrm{Si}$ and $\mathrm{SiC}$ at the high temperature and high switching frequency levels. GaN would have the smallest overall losses as regards $\mathrm{Si}$ and $\mathrm{SiC}$ transistors as the frequency rises. The temperature-dependent ratio $T_{d}$ is defined in equations (4) as:

$$
T_{d}=\frac{P_{\text {loss } 150}}{P_{\text {loss } 25}}
$$

where, $P_{\text {loss } 150}$ denotes the transistor losses at $150^{\circ} \mathrm{C}$ and $P_{\text {loss } 25}$ is the transistor losses at $25^{\circ} \mathrm{C}$ at $10 \mathrm{kHz}$ and $100 \mathrm{kHz}$ are shown in Fig. 8. The conduction loss estimation for the $\mathrm{GaN}$ device relies on the junction temperature of $T_{d}=2.8$, which is at least double the dependency of Si and $\mathrm{SiC}$ semiconductor devices. The $\mathrm{GaN}$ devices often demonstrate a high dependency on junction temperature $T_{d}=2$ for switching losses exceeding $\mathrm{Si}$ and $\mathrm{SiC}$ device's dependency.

Various losses in the GaN-based fly-back converter are shown in Fig. 10; it implies a reduction in power loss corresponding to a chosen efficiency point. The loss reduction for $\mathrm{GaN}$ and Si power converters is approximately equal for the operating frequency of $650 \mathrm{kHz}$ to $60 \mathrm{kHz}$, respectively. 


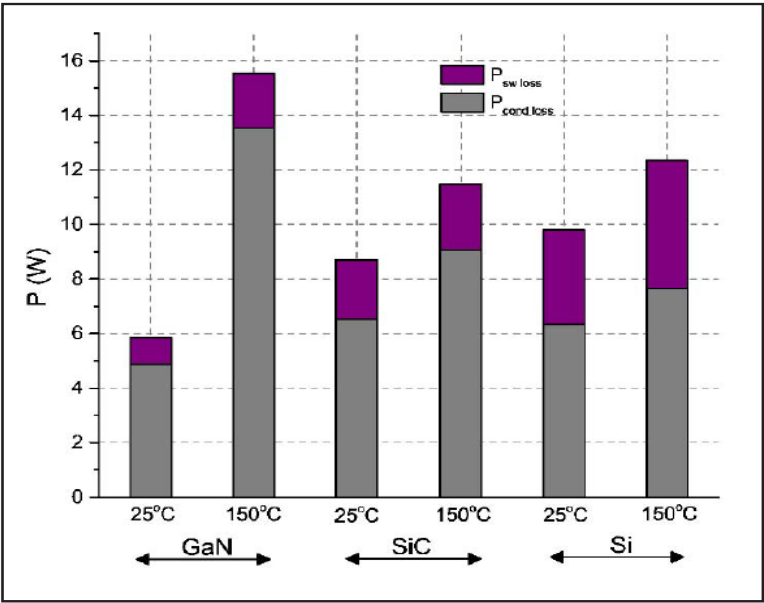

Fig 8. Switching loss and Conduction loss at $10 \mathrm{kHz}$ [24]

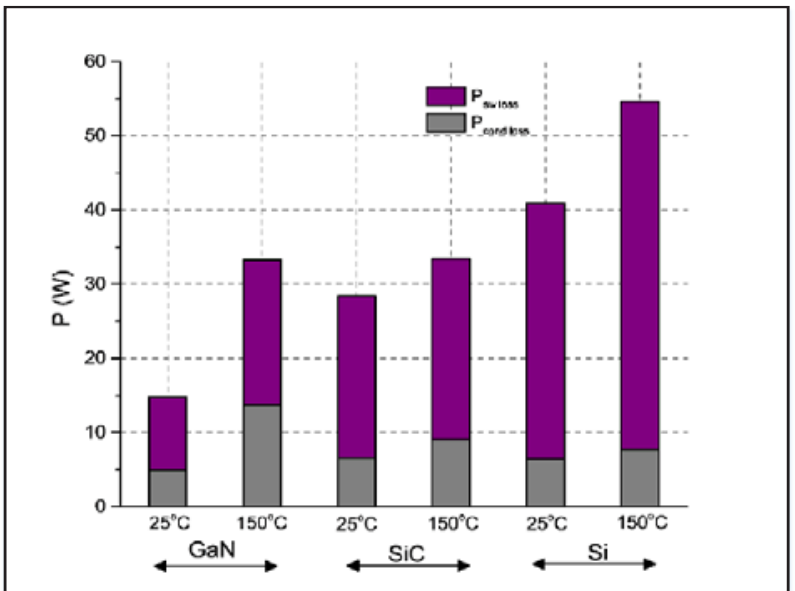

Fig 9. Switching loss and Conduction loss at $100 \mathrm{kHz}$ [24]

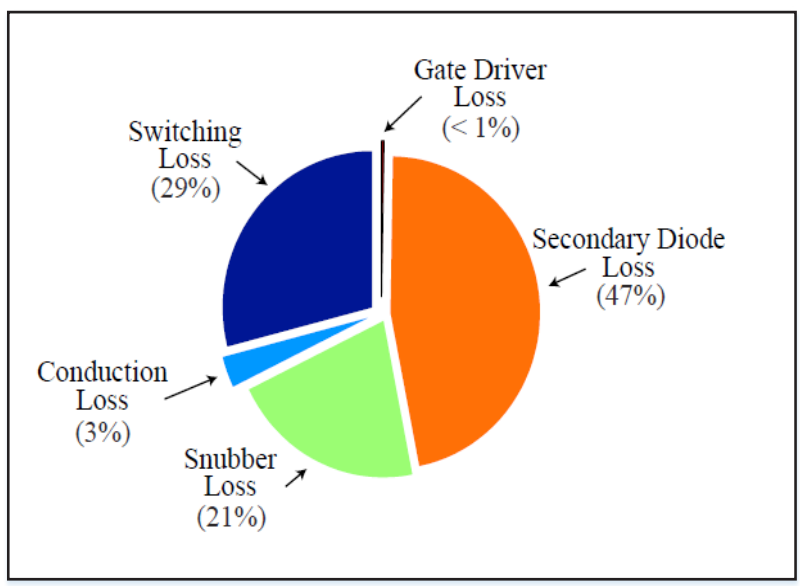

Fig 10. Different Losses in the GaN-based fly-back converter operating at a frequency of $650 \mathrm{kHz}$ [16] 


\subsection{Power Density Calculation}

The Si converter's total volume is around $185 \mathrm{~cm}^{3}$, while the GaN converter's total volume is $96 \mathrm{~cm}^{3}$ as shown in Fig. 11. The transformer with GaN converter requires significantly less implementation area compared to the Si converter. As a consequence, the overall volume of the $\mathrm{GaN}$ converter has been reduced.

The power density is assessed for GaN and Si power converter. The power density of the Si converter is $0.54 \mathrm{~W} / \mathrm{cm}^{3}$ whereas GaN-based converter's power density is $1.04 \mathrm{~W} / \mathrm{cm}^{3}$ [21]. The power density of the GaN converter is $92 \%$ higher than the Si converter. The comparison of volume distribution of flyback converter based on $\mathrm{GaN}$ and $\mathrm{Si}$ devices are shown in Fig. 11. It is visible from Fig. 11; the form factor of the power converter has been reduced by using a GaN. This leads to the high power converters like Vienna Rectifier can be made as small as possible for electric vehicle charging stations.

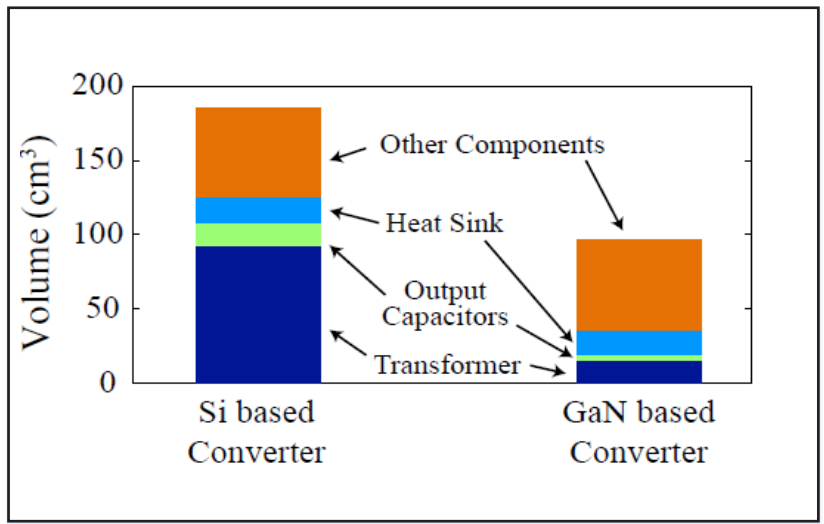

Fig 11. Evaluation of Volume Distribution for GaN and Si-based fly-back converter [23]

\section{Future Trends and its Applications}

Recent trends in GaN materials based on the converter on power density is shown in Fig. 12. It shows that the converter's size has been reduced when GaN-based materials are used for the converter design due to the high-power density. The converters will be designed using $\mathrm{GaN}$ material devices to reduce the overall system's size in the future.

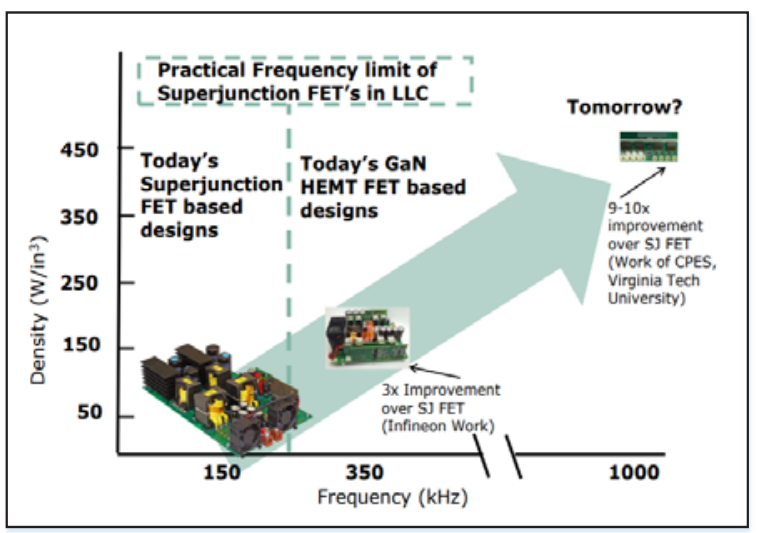


Fig 12. Recent Trends in GaN materials based converters on Power Density [14]

As reported in the previous sections, due to the advantages of the GaN materials, as shown in Fig.13, it is used for many applications such as wind power generation, electric vehicle charging stations, aircraft applications, data centers, and Telecommunication supplies. The applications of $\mathrm{GaN}$ devices for power converters are shown in Fig. 14.

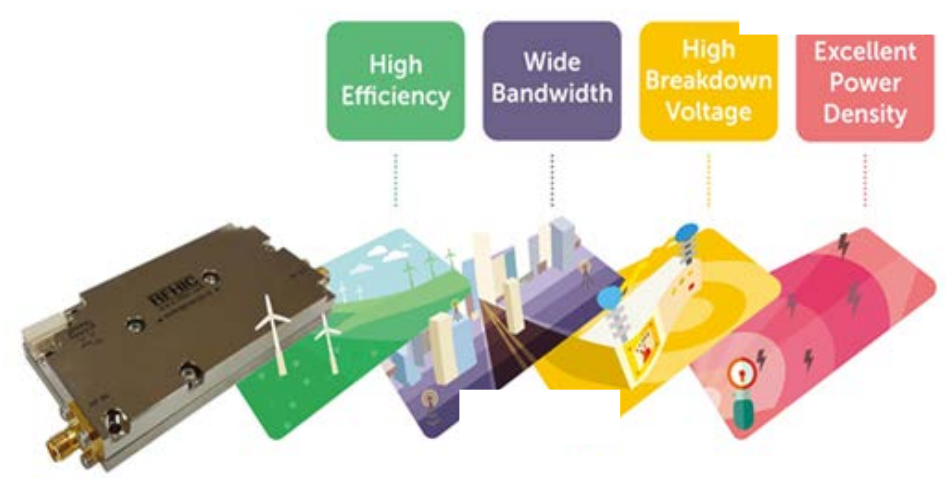

Fig 13. Benefits of GaN Devices based on its performances [18]

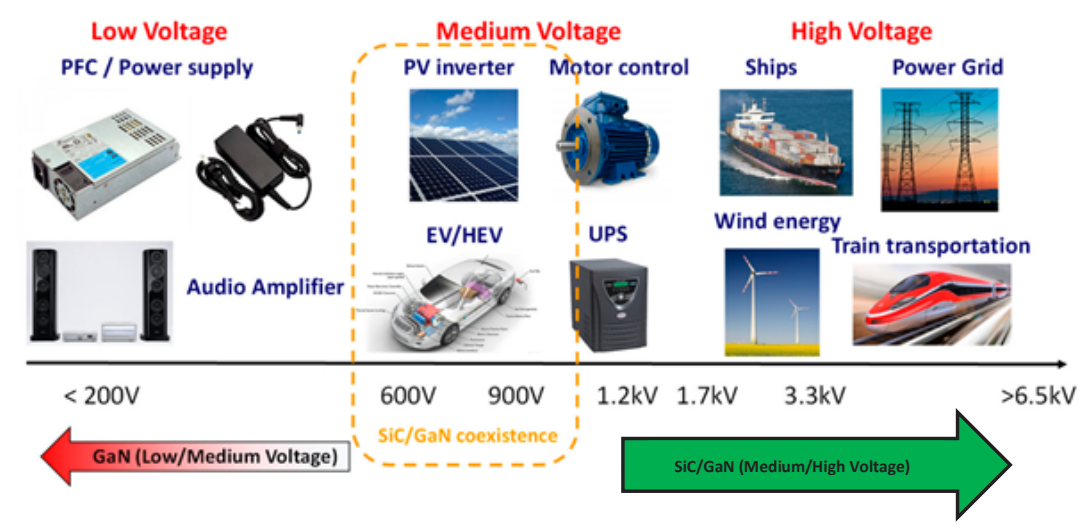

Fig 14. Applications of GaN Material Devices [18]

\section{Conclusions}

This paper has shown that the GaN device is an enabler for ultrahigh power density and highefficiency applications. It provides a comparison between $\mathrm{SiC}$ and $\mathrm{GaN}$ material devices in terms of the energy bandgap, carrier mobility, and saturation velocity dependency on temperature, which concludes that $\mathrm{GaN}$ devices have the switching frequency in the range of $100 \mathrm{MHz}$ and wide-bandgap. The applications of $\mathrm{GaN}$ devices can found in IoT, electric vehicle charging stations, More Electric Aircraft (MEA) applications, data centers, medical technology, unmanned aerial vehicles (UAV), autonomous cars, and drones to improve their performance. The GaN power device based Vienna Rectifier for the electric vehicle charging station has been proposed, which can reduce the harmonics in the input current has been reduced to less than 5\% to satisfy the IEEE-519 standard. The efficiency of the system has been improved, and the size of the system has been reduced by using GaN devices. 
Acknowledgement: This work is supported by Taylor's University under its TAYLOR'S RESEARCH SCHOLARSHIP programme through grant TUFR/2017/001/01.

\section{References}

1. R. Gowthamraj, C.V. Aravind, O.K.S. Prakash, AIP Conf. 2137 (2019)

2. G. Rajendran, C.A. Vaithilingam, K. Naidu, O.K.S. Prakash, SN Appl. Sci. 2, 583 (2020)

3. M. Hartmann, H. Ertl, J.W. Kolar, IEEE Trans. Pow. Elect. 27, 3137 (2012)

4. E.A. Jones, F.F. Wang, D. Costinett, IEEE J. Emer. Selec. Top. Power Elect. 4, 707 (2016)

5. E. Jones, F. Wang, D. Costinett, Z. Zhang, B. Guo, B. Liu, R. Ren, ECCE. 400 (2015).

6. F. Wang, Z. Zhang, T. Ericsen, R. Raju, R. Burgos, D. Boroyevich, Proceed. IEEE. 103, $2285(2015)$

7. Y. Zhang, M. Rodríguez, D. Maksimović, IEEE Trans. Power Elect. 31, 7926 (2016)

8. R. Nune, A. Anurag, S. Anand, Y.S. Chauhan, CPE-POWERENG. 347 (2016)

9. B. Hughes, Y.Y. Yoon, D.M. Zehnder, K.S. Boutros, CSICS. 1 (2011)

10. K. Shenai, IEEE Energy Technol. 1 (2013)

11. M. Hikita; M. Yanagihara; K. Nakazawa; H. Ueno; Y. Hirose; T. Ueda; Y. Uemoto; T. Tanaka; D. Ueda, T. Egawa, IEDM Technic. Digest. IEEE Int. Electron. Dev. Meet. 803 (2004)

12. Y. Uemoto, M. Hikita, H. Ueno, H. Matsuo, H. Ishida, M.Yanagihara, T. Ueda, T. Tanaka, D. Ueda, IEEE Trans. Elect. Dev. 54, 3393 (2007)

13. T. Ueda, T. Tanaka, D. Ueda, Intl. J. High-Speed Electron. Sys. 9, 145 (2009)

14. M.Yanagihara, Y.Uemoto, T. Ueda, T.Tanaka, D.Ueda, Appl. Mater. Sci. 206, 1221 (2009)

15. S.P. Oruganti, K. A. Vaithilingam, C. Rajendran, G. A, Ramasamy, Energies. 12, 3579 (2019)

16. Fan, H.Y. Phys. Rev. 82, 900 (1951)

17. S.S. Indira, C. A. Vaithilingam, K. Chong, R. Saidur, M. Faizal, S. Abubakar, S.Paiman, Sol. Energy. 201, 122 (2020)

18. Infineon Semiconductors Pvt. Ltd. Available online: https://www.infineon.com/cms/en/product/wide-band-gap-semiconductors-sic gan/silicon-carbide-sic/?redirId=130608\#! highlights. (Accessed on 05 December 2020)

19. A. M. S. Al-bayati, S. S. Alharbi, S. S. Alharbi, M. Matin, NAPS. 1 (2017)

20. Y. Zhu, Y. Han, IEEE. ECCE. 1 (2016)

21. IEEE Recommended Practice and Requirements for Harmonic Control in Electric Power Systems," in IEEE Std 519-2014 (Revision of IEEE Std 519-1992), 129 (2014)

22. N. Weste, D. Harris, A. Banerjee, A Circuit Sys. Perspec. 11, 739 (2005)

23. IND-08757 SERIES,” Available online: http://www.mpsindia.in/catalog/IND-08757/ (Accessed on 05 December 2020)

24. B. Al-Otaibi, P. Valizadeh, IEEE Trans. Dev. Mater. Reliab. 12, 547 (2012) 